\title{
Materiais compósitos a base de fibras da cana-de-açúcar e polímeros reciclados obtidos através da técnica de extrusão
}

\section{Composite materials based on fibers from sugar cane and recycled polymers obtained by extrusion technique}

\author{
Ezequiel Ligowski ${ }^{1}$, Benedito Cláudio dos Santos ${ }^{1}$ e Sérgio Toshio Fujiwara2* \\ 'Departamento de Química, Universidade Estadual do Centro Oeste - UNICENTRO, \\ Guarapuava, PR, Brasil \\ ${ }^{2}$ Departamento de Química, Universidade Estadual de Ponta Grossa - UEPG, \\ Ponta Grossa, PR, Brasil \\ *sergiofujiwara@gmail.com
}

\begin{abstract}
Resumo
Os compósitos obtidos a partir de fibras de bagaço de cana-de-açúcar e polímeros reciclados (PEAD e PS) foram obtidos através da técnica de extrusão a quente e o planejamento fatorial possibilitou a análise dos fatores que influenciam a obtenção dos compósitos. As amostras contendo polietileno de alta densidade (PEAD), tanto com 30\% como com 50\% de fibras, mostraram-se mais impermeáveis na presença da água que as amostras obtidas com poliestireno (PS). Os compósitos obtidos com PEAD apresentaram resistência à flexão com uma tensão máxima aplicada de $45 \mathrm{MPa}$ (50\% de fibra) e de $39 \mathrm{MPa}$ (30\% de fibra), esses valores são maiores que os apresentados pelos materiais obtidos com PS. Compósitos de polímeros e madeira (com 60\% de serragem) apresenta resistência à flexão de aproximadamente 32 MPa e o MDF apresenta uma resistência à flexão de $36 \mathrm{MPa}$, esses resultados mostram que os compósitos obtidos possuem uma boa resistência a flexão.
\end{abstract}

Palavras-chaves: compósitos, material reciclável, fibras naturais, técnica de extrusão.

\section{Abstract}

The composites were obtained through hot extrusion of a mixture of recycled polymers (HDPE and PS) and sugar cane fibers. The extrusion technique resulted in homogeneous materials with good mechanical properties and the factorial design allowed the analysis of the factors that influence the process. Samples containing high density polyethylene (HDPE), both with $30 \%$ and 50\% fibers were shown to be significantly more effective compared to polystyrene (PS) aiming at impermeability in the presence of water. The composites obtained with HDPE showed bending resistance, with $45 \mathrm{MPa}$ (50\% fiber) and 39MPa (30\% fiber) maximum applied stress, these values are higher than those presented in the composites obtained with PS. Composites of polymers and wood ( $60 \%$ of sawdust) provide bending resistance of about $32 \mathrm{MPa}$ and $\mathrm{MDF}$ has a bending resistance of $36 \mathrm{MPa}$, these results show that the compounds obtained have a good bending resistance.

Keywords: composites, recyclable polymers, natural fibers, extrusion technique.

\section{Introdução}

A conscientização da sociedade firmada no conceito de desenvolvimento sustentável exige a produção de materiais ecológicos de baixo custo e consumo de energia reduzida. Buscam-se produtos capazes de satisfazer requisitos fundamentais como a conservação de recursos naturais não renováveis e a manutenção de um ambiente saudável. Os materiais compósitos, produzidos com fibras naturais, destacam-se neste conceito ${ }^{[1,2]}$.
Compósitos são materiais formados pela combinação de dois ou mais diferentes materiais, produzindo propriedades únicas e sinergísticas, diferentes daquelas de seus componentes individuais ${ }^{[3]}$. Eles são, portanto, constituídos de duas fases: a matriz e o elemento de reforço, e são desenvolvidos para otimizar os pontos fortes de cada uma das fases. O material matriz é o que confere estrutura ao material compósito, preenchendo os espaços vazios que 
ficam entre os materiais reforços e mantendo-os em suas posições relativas. Os materiais reforços são os que realçam propriedades mecânicas, eletromagnéticas ou químicas do material compósito como um todo ${ }^{[4]}$. Os termoplásticos são materias poliméricos sintéticos, que, quando sujeitos à ação de calor, facilmente se deformam podendo ser remodelados e novamente solidificados a uma nova estrutura. Se apresentam como uma excelente matriz polimérica devido a sua alta abundância, podendo o mesmo ser reciclado. As interações intermoleculares presentes nos materiais termoplásticos são rompidas no processo de fundição, permitindo a adição de reforços que estabelecem interações com o material termoplástico no processo de resfriamento, obtendo-se assim o material compósito ${ }^{[4]}$.

Compósitos de matriz polimérica com diversos tipos de cargas, em particular os reforçados por fibras, têm recebido muita atenção desde a segunda guerra mundial. A importância desses compósitos é muito grande, sobretudo no campo de aplicação e desenvolvimento de peças automobilísticas, esportivas, aeronáuticas, moveleiras e na indústria da construção civil. Os compósitos poliméricos reforçados por fibras vegetais também têm sido alvo de grande interesse acadêmico e industrial por substituírem, geralmente com vantagens de custo e leveza, as peças feitas de compósitos poliméricos convencionais ou mesmo peças feitas inteiramente de plásticos. Esses compósitos inserem-se na política de aproveitamento de recursos renováveis, menos agressivos e tóxicos, visto que, as matérias primas de origem vegetal, tais como: óleos, fibras, corantes, etc., além de, serem oriundas de fontes renováveis, atendem aos requisitos de biodegradabilidade e preservação do meio ambiente durante todo o seu ciclo de vida ${ }^{[1,2,5-11]}$. Esses materiais estão sendo empregados cada vez mais em substituição aos tradicionais (monolíticos) cujas características individuais não atendem às crescentes exigências de melhor desempenho, durabilidade e economia, apresentando várias vantagens em sua utilização, tais como: elevada resistência e rigidez específica, baixa densidade e resistência à corrosão $0^{[4]}$.

Historicamente a cana-de-açúcar é um dos principais produtos agrícolas do Brasil, sendo cultivada desde a época da colonização. Devido à grandeza dos números do setor sucroalcooleiro no Brasil, não se pode tratar a cana-de-açúcar, apenas como mais um produto, mas sim como o principal tipo de biomassa energética, base para todo o agronegócio sucroalcooleiro, representado por 350 indústrias de açúcar e álcool e 1.000.000 empregos diretos e indiretos em todo o país ${ }^{[5]}$.

O bagaço e a palha de cana-de-açúcar são materiais lignocelulósicos gerados em grandes proporções, constituídos principalmente por celulose, hemicelulose e lignina. A celulose, principal componente dessas fibras vegetais, é um homopolímero linear composto de unidades de anidroglicose, as quais são ligadas entre si através de ligações $\beta$-(1-4)-glicosídicas, e possui estrutura fibrilar e módulo de elasticidade relativamente alto. A maior parte desse resíduo agroindustrial é utilizado na própria indústria alcooleira para geração de energia, o excedente pode ser utilizado em outras aplicações mais nobres, por exemplo como reforço em materiais compósitos ${ }^{[5]}$.
O processo de extrusão é um dos métodos mais adequados para a obtenção de materiais compósitos, ele consiste basicamente numa conversão de matéria-prima apropriada em um determinado produto com produção de forma semi-contínua onde o material é forçado através de uma matriz adquirindo assim forma pré-determinada sob condições controladas. A extrusora é um equipamento capaz de fornecer pressão contínua e uniforme ao material extrudado e também pode possuir várias zonas de aquecimento ${ }^{[7,10,11]}$.

\section{Parte Experimental}

\subsection{Seleção da matéria-prima}

Após a realização de testes preliminares com vários polímeros reciclados, os polímeros escolhidos para a obtenção dos compósitos foram: o polietileno de alta densidade (PEAD) em forma de granulados e o poliestireno cristal (PS) obtido através de copos descartáveis. Ambos os polímeros foram obtidos na empresa Replastik, localizada na rodovia BR 277, Km 350, Guarapuava, Paraná. Esses polímeros foram selecionados devido a suas diferentes propriedades, para uma melhor interpretação dos resultados obtidos no planejamento fatorial.

Como reforço do materiais compósitos utilizou-se fibras vegetais de bagaço de cana-de-açúcar coletadas na usina Vale do Ivaí localizada em São Pedro do Ivaí - PR. Devido à abundância dessas fibras no país e seu baixo custo, sua utilização na preparação dos compósitos mostrou-se de grande interesse. As fibras de bagaço de cana-de-açúcar foram secas em estufas a $100^{\circ} \mathrm{C}$ durante o tempo de 12 horas, para retirada de toda a umidade das fibras. Em seguida as fibras foram trituradas em um moinho de facas e peneiradas em uma peneira de 16 mesh para a homogeneização da granulometria das fibras, esse processo se faz necessário para uma melhor incorporação do reforço na matriz polimérica para fabricação dos compósitos propostos.

\subsection{Moagem da matéria-prima}

Após coleta e seleção dos materiais utilizados, com exceção do polietileno de alta densidade que já se encontrava em granulados, tanto as fibras de bagaço de cana-de-açúcar como os copos descartáveis reciclados de poliestireno foram devidamente triturados em um moinho triturador de facas. Esse processo serve para diminuir a granulometria dos componentes da mistura, melhorando a homogeneidade da mistura e facilitando a passagem pela extrusora.

\subsection{Matriz de planejamento fatorial de dois níveis}

Para a obtenção dos compósitos realizou-se um planejamento fatorial $2^{2}$, no qual foram analisados dois fatores em dois níveis diferentes. Os fatores escolhidos foram o tipo de polímero utilizado e a proporção de fibras na confecção de cada amostra. A Tabela 1 mostra o planejamento fatorial $2^{2}$ realizado.

\subsection{Obtenção dos compósitos}

Os compósitos foram confeccionados conforme os ensaios proposto na Tabela 1. Os componentes da mistura foram pesados e passados por um misturador termocinético, 
Tabela 1. Planejamento Fatorial $2^{3}$ para otimização do processamento.

\begin{tabular}{ccc}
\hline Variável & Nível (-) & Nível (+) \\
\hline $\begin{array}{c}\text { Tipo de polímero } \\
- \text { TP }\end{array}$ & PEAD & PS \\
$\begin{array}{c}\text { Proporção de fibras } \\
- \text { PF (\%) }\end{array}$ & 30 & 50 \\
\hline & Variável & \\
\hline Ensaios & TP & PF \\
\hline 1 & - & - \\
2 & + & - \\
3 & - & + \\
4 & + & + \\
\hline
\end{tabular}

este equipamento tem por função homogeneizar e aglutinar o material, deixando os aglomerados mais densos para maior fluidez no processamento. Completada a homogeneização da mistura, chega-se na parte mais importante na preparação do compósito, a extrusão. Neste processo ocorre a fusão no polímero e a mistura com as fibras na passagem do material por uma monorosca. A velocidade de rotação da rosca utilizada na extrusora foi de 100 RPM, e as temperaturas de extrusão da zona 1 (alimentação) em $160^{\circ} \mathrm{C}$, da zona 2 (compressão) $175^{\circ} \mathrm{C}$, da zona 3 (dosificação) $190^{\circ} \mathrm{C}$ e do cabeçote em $190^{\circ} \mathrm{C}$.

No processo de extrusão procurou-se trabalhar com uma temperatura suportável aos constituintes da mistura para que os mesmos não sofressem degradação. As temperaturas de trabalho escolhidas em cada zona de passagem da extrusão foi fixada levando em consideração a temperatura de fusão dos constituintes e a temperatura de combustão das fibras para que as mesmas não queimassem.

Utilizou-se para a preparação destes compósitos uma mini-extrusora GPE $40 \mathrm{~mm}$ adaptada para o processamento de materiais compósitos, composta por estrutura em chapa de aço carbono dobrado, canhão e rosca de $40 \mathrm{~mm}$ de diâmetro por $900 \mathrm{~mm}$ de comprimento, motor de $0,5 \mathrm{CV}$, painel para controle de temperatura com 3 zonas de aquecimento e resistências elétricas de $220 \mathrm{v}$.

Com os corpos de prova ainda quentes e moldáveis após extrusão, foi feito a prensagem do material compósito em moldes preparados de acordo com as devidas normas técnicas. Utilizou-se uma prensa hidráulica, marca Bovenau, com capacidade máxima de 15 toneladas de pressão. As amostras compósitas foram prensadas utilizando-se uma pressão padrão de 10 toneladas, num tempo de prensagem de 90 segundos.

Os corpos de prova foram confeccionados de acordo com as normas D-1037 (ASTM, 1995) e ASTM D790 que são as normas estabelecidas para os ensaios de absorção de água (umidade) e flexão estática.

\subsection{Realização de testes físicos}

Os materiais compósitos produzidos foram submetidos a testes de umidade e flexão estática, e os resultados obtidos foram analisados através de um planejamento fatorial.

\subsubsection{Teste de umidade}

Na realização deste teste, foram preparados corpos de prova com as dimensões de $0,5 \mathrm{~mm} \times 5 \mathrm{~cm} \times 5 \mathrm{~cm}$, submersos em água à temperatura constante de $20^{\circ} \mathrm{C}$. As amostras tiveram suas massas iniciais anotadas para posterior cálculo de variação na umidade, sendo feitas em quadruplicata para que os dados encontrados neste teste ganhassem uma confiabilidade maior nos resultados. O material compósito ficou disposto horizontalmente através de uma grade onde cada amostra foi colocada entre os espaços que separam este material, tendo com isso todos os corpos de prova submersos a mesma profundidade com separação praticamente igual entre eles.

O peso das amostras condicionadas em uma câmara climatizada foi feito após um período de 2 e 24 horas de submersão em água. $\mathrm{O}$ teste de umidade realizado seguiu a norma norte-americana D-1037 (ASTM, 1995). Esses ensaios foram realizados no laboratório de materiais da UNICENTRO (Universidade Estadual do Centro-Oeste) utilizando um banho termostático onde as amostras ficaram imergidas.

\subsubsection{Teste de flexão estática}

Para a realização deste teste foram confeccionadas amostras de seção retangulares de $0,6 \mathrm{~mm} \times 6 \mathrm{~cm} \times 2 \mathrm{~cm}$ obtidas através do processo de prensagem como estabelece a norma ASTM D790 para resistência à flexão estática adaptada para o ensaio mecânico de três pontos. Neste ensaio dois pontos são mantidos fixos e o terceiro ponto, movimenta-se com esforço axial no centro do corpo de prova. A amostra é testada a uma taxa de tensão de $2,8 \mathrm{~mm} / \mathrm{min}$, até que ocorra a ruptura, ou seja, promovida uma tensão máxima de $5 \%$. Esses ensaios foram realizados no laboratório de engenharia de materiais da UEPG (Universidade Estadual de Ponta Grossa) utilizando-se de equipamento universal EMIC DL 30.000, com transferência de força e velocidade de teste controlada.

\section{Resultados e Discussão}

\subsection{Preparação dos materiais compósitos}

Testes preliminares indicaram que determinados polímeros reciclados eram inadequados para a fabricação dos compósitos com fibras de bagaço de cana-de-açúcar, pois a fusão destes termoplásticos ocorre em temperaturas acima de $200^{\circ} \mathrm{C}$, e desta forma, a incorporação de reforço fibroso se torna comprometida, ocorrendo degradação térmica em sua estrutura, quando utilizadas altas temperaturas de processamento. Verificou-se também a necessidade de utilização do bagaço seco ao invés dele "in natura". A fibra verde apresenta grande quantidade de umidade e de compostos voláteis, o quê dificulta o processamento por extrusão, pois o excesso de gases que se forma não é liberado na zona de degasagem do equipamento e acaba incorporado ao compósito, formando espaços vazios que prejudicam as características estruturais do material. $\mathrm{O}$ resíduo da cana-de-açúcar apresenta mínima quantidade de açúcar em sua composição, visto que com as tecnologias avançadas, a extração do caldo é praticamente máxima nas usinas sucroalcooleiras. $\mathrm{O}$ açúcar tem sua temperatura de fusão e decomposição entre $160^{\circ} \mathrm{C}$ e $190^{\circ} \mathrm{C}$, portanto, a pequena quantidade presente no bagaço de cana-de-açúcar é eliminada por volatilização ou praticamente desconsiderada como um aditivo reforçante em compósitos estruturais.

As amostras compósitas foram preparadas, utilizando $30 \mathrm{~g}$ das matérias-primas necessárias nas devidas proporções 
de estudo. Em todas elas trabalhou-se com um excesso de $5 \%$, ou seja, $1,5 \mathrm{~g}$, que foi considerado devido a perdas tanto na mistura dos componentes como no processo posterior de extrusão.

Os compósitos foram confeccionados segundo um planejamento fatorial $2^{2}$, no qual foram analisados dois fatores em dois níveis diferentes, com a finalidade de verificar a influência da proporção da fibra e do tipo de polímero nas propriedades dos compósitos obtidos.

Os polímeros escolhidos para a obtenção dos compósitos foram: o polietileno de alta densidade (PEAD) em forma de granulados e o poliestireno cristal (PS) obtido através de copos descartáveis. Esses polímeros foram selecionados devido a suas diferentes propriedades, para uma melhor interpretação dos resultados obtidos no planejamento fatorial. As proporções de fibras que foram estudadas como reforço nos dois tipos de polímeros usados tiveram margens de $20 \%$ de diferença ( $30 \%$ e $50 \%$ ), sendo adotadas estas quantidades pois em testes preliminares observou-se excelentes resultados para serem comparados e ótimo processamento nos compósitos formulados, permitindo uma melhor transparência nas análises e interpretação dos dados.

\subsection{Absorção de água}

As amostras para absorção de água foram devidamente confeccionadas seguindo a norma norte-americana D-1037 (ASTM, 1995), igualando as dimensões de todas elas em $0,5 \mathrm{~mm} \times 5 \mathrm{~cm} \times 5 \mathrm{~cm}$. Os ensaios foram realizados em quadruplicata, e os corpos de prova foram obtidos com muito cuidado, isto é, todos os corpos de prova foram confeccionados, seguindo a risca, o mesmo procedimento. Outra preocupação deste procedimento foi a de utilizar água destilada, eliminando possíveis interferentes presentes no líquido e manter a temperatura de $20^{\circ} \mathrm{C}$ constante na realização do teste. A não inclusão de variabilidade no processo torna os possíveis erros que venham a aparecer menores, e com isso os efeitos vistos mais significativos ${ }^{[12]}$.

Outro procedimento adotado confecção das amostras foi à ordem aleatória de realização dos ensaios. A aleatorização dos ensaios ajuda a impedir que fatores indesejáveis (luminosidade, clima do dia, etc), contaminem os efeitos que queremos investigar. Sorteando a realização dos ensaios a probabilidade de um desses fatores indesejáveis afetar uma resposta é a mesma para todas as respostas, dessa forma sua atuação ficará diluída e a confiabilidade nos resultados será mais efetiva. A numeração nos ensaios é apenas uma forma conveniente de identificar as várias combinações de níveis, e, portanto, essa ordem nada tem a ver com a ordem em que os experimentos foram realizados ${ }^{[12]}$.
Os valores médios em porcentagem de absorção de água nos polímeros com suas devidas proporções em fibra após 2 e 24 horas de imersão em água encontram-se na Tabela 2.

De acordo com a Tabela 2 observou-se que a maior proporção de fibra aumenta a absorção de água nos compósitos, sendo isto esperado devido às características higroscópicas que as fibras lignocelulósicas apresentam. Verificou-se que utilizando o polietileno de alta densidade ao invés do poliestireno, foram obtidos materiais com melhores resultados quando submetido a testes de umidade.

O efeito principal do tipo de polímero (TP) é, por definição, a média dos efeitos do tipo de polímero nos dois níveis de proporção de fibra. Para representar esse efeito, e sendo $\bar{y}_{i}$ a resposta média observada no i-ésimo ensaio, pode-se então escrever ${ }^{[12]}$ (Equação 1):

$$
T P=\frac{\left(\bar{y}_{2}-\bar{y}_{1}\right)+\left(\bar{y}_{4}-\bar{y}_{3}\right)}{2}
$$

Para representação das proporções de fibras (PF), no cálculo do efeito principal, em nível superior e inferior, pode-se escrever ${ }^{[12]}$ (Equação 2):

$$
P F=\bar{y}_{+}-\bar{y}_{-}=\frac{\left(\bar{y}_{3}+\bar{y}_{4}\right)}{2}-\frac{\left(\bar{y}_{1}+\bar{y}_{2}\right)}{2}
$$

Quando o efeito de uma variável depende do nível de outra, como no caso desse planejamento, se diz que as duas variáveis interagem, e assim é possível calcular o valor do efeito de interação entre elas. Usando-se TP x PF para representar esse efeito tem-se ${ }^{[12]}$ (Equação 3):

$$
T P \times P F=\frac{\left(\bar{y}_{4}-\bar{y}_{3}\right)}{2}-\frac{\left(\bar{y}_{2}-\bar{y}_{1}\right)}{2}=\frac{\left(\bar{y}_{1}+\bar{y}_{4}\right)}{2}-\frac{\left(\bar{y}_{2}+\bar{y}_{3}\right)}{2}
$$

O cálculo dos efeitos das variáveis foi realizado e os valores obtidos são apresentados na Tabela 3 .

Com os cálculos dos efeitos principais algumas conclusões importantes foram obtidos neste estudo. Quando o polietileno de alta densidade é substituído pelo poliestireno, notou-se um aumento na absorção de água de $0,14 \%$ em 2 horas e de $1,41 \%$ em 24 horas, portanto, conclui-se que a utilização do PS não é interessante para confecção de compósitos que possam estar constantemente expostos a umidade. Outro dado importante, nos diz que quando aumenta-se a proporção de fibras no compósito, observa-se a higroscopicidade aumentar $0,59 \%$ em 2 horas de imersão e $1,5 \%$ em 24 horas de imersão, mostrando que a maior quantidade de cargas fibrosas reforçantes no compósito podem auxiliar em alguns ensaios mecânicos, mas não nos testes físicos de absorção de água.

Quando foram comparados os valores médios de teores de absorção de água encontrados em todas as amostras com

Tabela 2. Resultados encontrados nos testes de absorção de água após 2 e 24 horas.

\begin{tabular}{ccccc}
\hline Ensaio & TP & PF (\%) & AB (\%) em $\mathbf{~ h}$ & AB (\%) em 24h \\
\hline 1 & PEAD & 30 & $0,66 \pm 0,02$ & $1,46 \pm 0,03$ \\
2 & PS & 30 & $0,74 \pm 0,02$ & $1,94 \pm 0,03$ \\
3 & PEAD & 50 & $1,18 \pm 0,02$ & $2,03 \pm 0,03$ \\
4 & PS & 50 & $1,39 \pm 0,02$ & $4,36 \pm 0,03$ \\
\hline
\end{tabular}

Onde: $\mathrm{AB}=$ Absorção média de água em $\%$ de massa, $\mathrm{TP}=$ Tipo de Polímero, $\mathrm{PF}=$ Proporção de Fibras. 
os valores relatados de chapas aglomeradas, briquetes de madeira, cavacos e cascas de madeira, os resultados obtidos no presente trabalho mostram-se altamente significativos ficando bem abaixo dos limites estabelecidos pelas normas. Estes baixos valores obtidos no ensaio de absorção de água, mostra que o compósito pode ser utilizado como um material alternativo a madeira e seus derivados nos setores da construção civil e indústria moveleira. As chapas aglomeradas, por exemplo, oscilam com valores que vão de $41,05 \%$ a $81,48 \%$ de absorção de água, os briquetes de madeira vão de 5 a $10 \%$, os cavacos de madeira de 20 a $30 \%$ e as cascas chegam a absorver de 40 a $60 \%$ em água. $\mathrm{O}$ valor mais próximo encontrado na literatura é o do MDF (Medium Density Fiberboard) que absorve aproximadamente $3 \%$ de água em 2 horas. Um compósito de polipropileno com $25 \%$ de bagaço de cana-de-açúcar feito por compressão a quente apresentou uma absorção de água de aproximadamente $6 \%{ }^{[13,14]}$.

Concluí-se, portanto, que os compósitos obtidos absorvem pouca água e estão aprovados em relação ao critério de absorção de água.

\subsection{Flexão estática}

As amostras para o teste de flexão estática foram devidamente confeccionadas seguindo a norma norte-americana ASTM D790, igualando sua dimensões em $0,6 \mathrm{~mm}$ de espessura, $6 \mathrm{~cm}$ de comprimento por $2 \mathrm{~cm}$ de largura. As amostras que foram feitas em quintuplicada e os ensaios de flexão foram realizadas sob a velocidade de $2,8 \mathrm{~mm} / \mathrm{min} \mathrm{e}$ o método de análise adotado foi o de três pontos de apoio

Tabela 3. Valores dos efeitos dos fatores principais e da interação entre eles, para ensaios de absorção de água em 2 e 24 horas de exposição.

\begin{tabular}{ccc}
\hline Efeitos principais & $\begin{array}{c}\text { Valores dos efeitos } \\
(\%)\end{array}$ & $\begin{array}{c}\text { Valores dos efeitos } \\
(\%)\end{array}$ \\
& $\begin{array}{c}(\mathbf{2} \text { horas de } \\
\text { imersão) }\end{array}$ & $\begin{array}{c}\text { (24 horas de } \\
\text { imersão) }\end{array}$ \\
\hline TP & 0,14 & 1,41 \\
PF & 0,59 & 1,50 \\
TP $\times$ PF & 0,06 & 0,92 \\
\hline
\end{tabular}

Tabela 4. Resultados encontrados nos testes de flexão estática.

\begin{tabular}{cccc}
\hline Ensaio & TP & PF (\%) & $\begin{array}{c}\text { Resistência a } \\
\text { Flexão (MPa) }\end{array}$ \\
\hline 1 & PEAD & 30 & $39 \pm 1$ \\
2 & PS & 30 & $33 \pm 1$ \\
3 & PEAD & 50 & $45 \pm 1$ \\
4 & PS & 50 & $38 \pm 1$ \\
\hline
\end{tabular}

Tabela 5. Valores dos efeitos dos fatores principais e da interação entre eles.

\begin{tabular}{cc}
\hline Efeitos principais & Valores dos efeitos (MPa) \\
\hline TP & $-6,5$ \\
PF & 5,5 \\
TP $\times$ PF & $-0,5$ \\
\hline
\end{tabular}

com carregamento no ponto médio. Os dados dos testes serão apresentados na Tabela 4.

Observa-se que a maior proporção de fibra tornou os compósitos mais resistentes à flexão, conferindo ao compósito uma maior rigidez, esse fato é observado tanto para o PEAD como para o PS. O aumento do teor de fibras proporciona uma melhor distribuição das tensões e o material passa a apresentar maior resistência a flexão ${ }^{[15,16]}$.

O cálculo dos efeitos das variáveis foi realizado e os valores obtidos são apresentados na Tabela 5 .

Observa-se que tanto o TP como a PF são determinantes para a resistência a flexão dos compósitos preparados.

Os compósitos obtidos com PEAD apresentaram resistência à flexão com uma força aplicada de $45 \mathrm{MPa}$ ( $50 \%$ de fibra) e de $39 \mathrm{MPa}$ ( $30 \%$ de fibra), esses valores são maiores que os apresentados pelos compósitos de polímeros e madeira (com $60 \%$ de serragem) que é de aproximadamente $32 \mathrm{MPa}$ e o MDF que apresenta uma resistência a força de $36 \mathrm{MPa}^{[1]}$, mostrando que os compósitos obtidos possuem boa resistência a flexão.

\section{Conclusões}

A proposta de obtenção de materiais compósitos através de materiais reciclados foi realizada com sucesso, no trabalho realizado foram obtidos compósitos com boas propriedades mecânicas utilizando fibras de bagaço de cana-de-açúcar e polímeros reciclados (PEAD e PS).

A técnica de extrusão permitiu a obtenção de materiais homogêneos com boas propriedades mecânicas e o planejamento fatorial possibilitou a análise dos fatores que influenciam a obtenção dos compósitos.

As amostras contendo polietileno de alta densidade (PEAD) tanto com $30 \%$ como com $50 \%$ mostraram-se claramente mais eficazes quando relacionadas ao objetivo de serem mais impermeáveis na presença de água. Quando comparamos os valores médios de teores de umidade encontrados em todas as amostras com chapas aglomeradas, briquetes de madeira,cavacos e cascas de madeira por exemplo, os resultados obtidos no presente trabalho mostram-se altamente significativos ficando bem abaixo dos limites estabelecidos pelas normas. As chapas aglomeradas, por exemplo, oscilam com valores que vão de $41,05 \%$ a $81,48 \%$ de absorção de água, os briquetes de madeira vão de 5 a $10 \%$ de teor de umidade, os cavacos de madeira de 20 a $30 \%$ em umidade e as cascas chegam a absorver de 40 a 60\% em água. Os compósitos obtidos com $\mathrm{PEAD}$ apresentaram resistência à flexão com uma força aplicada de $45 \mathrm{MPa}$ ( $50 \%$ de fibra) e de $39 \mathrm{MPa}$ ( $30 \%$ de fibra), esses valores são maiores que os apresentados pelos compósitos de polímeros e madeira (com 60\% de serragem) que é de aproximadamente $32 \mathrm{MPa}$ e o MDF que apresenta uma resistência a força de $36 \mathrm{MPa}^{[1]}$, mostrando que os compósitos obtidos possuem boa resistência a flexão.

\section{Agradecimentos}

Os autores agradecem a Fundação Araucária pelo financiamento recebido. 


\section{Referências}

1. Hillig, E., Freire, E., Carvalho, G. A., Schneider, V. E., \& Pocai, K. (2006). Modelagem de misturas na fabricação de compósitos polímero-fibra, utilizando polietileno e serragem de Pinus sp. Ciência Florestal, 16(1), 343-351.

2. Neto, J. R. A., Carvalho, L. H., \& Araújo, E. M. (2007). Influência da adição de uma carga nanoparticulada no desempenho de compósitos poliuretano/fibra de juta. Polímeros, 17(1), 1015. http://dx.doi.org/10.1590/S0104-14282007000100006.

3. Zarbin, A. J. G. (2007). Química de (nano)materiais. Química Nova, 30(6), 1469-1479. http://dx.doi.org/10.1590/S010040422007000600016 .

4. Schwartz, M. M. (1997). Composite Materials: Processing, Fabrication and Applications (Vol. 2). New Jersey: Prentice Hall.

5. Luz, S., Gonçalves, A. R., \& Del'Arco, A. P. Jr (2006) Microestrutura e propriedades mecânicas de compósitos de polipropileno reforçado com celulose de bagaço e palha de cana. Revista Matéria, 11(2), 101-110. http://dx.doi.org/10.1590/ S1517-70762006000200004

6. Nohara, L. B., Kawamoto, A. M., Takahashi, M. F. K., Wills, M., Nohara, E. L., \& Rezende, M. C. (2004). Síntese de um poli (ácido âmico) para aplicação como interfase em compósitos termoplásticos de alto desempenho. Polímeros, 14(2), 122 128. http://dx.doi.org/10.1590/S0104-14282004000200016.

7. Vianna, W. L., Correa, C. A., \& Razzino, C. A. (2004). Efeitos do tipo de poliestireno de alto impacto nas propriedades de compósitos termoplásticos com farinha de resíduo de madeira. Polímeros, 14(5), 339-348. http://dx.doi.org/10.1590/S010414282004000500012.

8. Rodolfo, A., Jr., \& John, V. M. (2006). Desenvolvimento de PVC reforçado com resíduos de Pinus para substituir madeira convencional em diversas aplicações. Polímeros, 16(1), 1-11. http://dx.doi.org/10.1590/S0104-14282006000100005.

9. Vallejos, M. E., Curvelo, A. A. S., Teixeira, E. M., Mendes, F. M., Carvalho, A. J. F., Felissia, F. E., \& Area, M. C. (2011) Composite materials of thermoplastic starch and fibers from the ethanol-water fractionation of bagasse. Industrial Crops and Products, 33(3), 739-746. http://dx.doi.org/10.1016/j. indcrop.2011.01.014.

10. Mulinari, D. R., Voorwald, H. J. C., Cioffi, M. O. H., Silva, M. L. C. P., Cruz, T. G., \& Saron, C. (2009). Sugarcane bagasse cellulose/HDPE composites obtained by extrusion. Composites Science and Technology, 69(2), 214-219. http:// dx.doi.org/10.1016/j.compscitech.2008.10.006.

11. Guimarães, J. L., Wypych, F., Saul, C. K., Ramos, L. P., \& Satyanarayana, K. G. (2010). Studies of the processing and characterization of corn starch and its composites with banana and sugarcane fibers from Brazil. Carbohydrate Polymers, 80(1), 130-138. http://dx.doi.org/10.1016/j.carbpol.2009.11.002.

12. Neto, B. B., Scarminio, I. S., \& Bruns, R. E. (2002). Como fazer experimentos: pesquisa e desenvolvimento na ciência e na indústria (2nd ed.). Campinas: Ed UNICAMP.

13. Pauleski, D. T., Haselein, C. R., Santini, E. J., \& Rizzatti, E. (2007). Características de compósitos laminados manufaturados com polietileno de alta densidade (PEAD) e diferentes proporções de casca de arroz e partículas de madeira. Ciência Florestal, 17(2), 157-170.

14. Caraschi, J. C., Leão, A. L., \& Chamma, P. V. C. (2009). Avaliação de painéis produzidos a partir de resíduos sólidos para aplicação na arquitetura. Polímeros, 19(1), 47-53. http:// dx.doi.org/10.1590/S0104-14282009000100012.

15. Ishizaki, M. H., Visconte, L. L. Y., Furtado, C. R. G., Leite, M. C. A. M., \& Leblanc, J. L. (2006). Caracterização mecânica e morfológica de compósitos de polipropileno e fibras de coco verde: influência do teor de fibra e das condições de mistura. Polímeros, 16(3), 182-186. http://dx.doi.org/10.1590/S010414282006000300006 .

16. Ibrahim, M., Dufresne, A., El-Zawawy, W. K., \& Agblevor, F. (2010). Banana fibers and microfibrils as lignocellulosic reinforcements in polymer composites. Carbohydrate Polymers, 81(4), 811-819. http://dx.doi.org/10.1016/j.carbpol.2010.03.057.

Enviado: Nov. 11, 2013

Reenviado: Jul. 04, 2014 Aceito: Ago. 04, 2014 